\title{
LA SUBJETIVIDAD EN KANT Y LEVINAS: AUTONOMÍA Y PASIVIDAD FRENTE A LA ALTERIDAD
}

Subjectivity in Kant and Levinas: Autonomy and passivity in front of alterity

\author{
DAVID MARTÍNEZROJAS \\ Universidad San Sebastián (Chile) \\ Universidad Bernardo O'Higgins (Chile) \\ david.martinez@uss.cl
}

\section{Resumen}

El artículo analiza la noción de subjetividad de Levinas y se examinan sus posibles vínculos con la noción desarrollada por Kant, entendida esta última como una visión ejemplar de la explicación moderna acerca de la subjetividad. Se discute la posición de Hofmeyr, quien argumenta que hubo un cambio paradigmático entre un "Levinas temprano" centrado en la libertad de tipo kantiana, y un "Levinas posterior" centrado en la pasividad y que trasciende a Kant. El artículo muestra que la subjetividad en Levinas nunca puede entenderse como actividad en el sentido kantiano.

Palabras clave: Subjetividad; Levinas; Kant; alteridad; actividad-pasividad; vida del goce; substitución

\section{Abstract:}

The article analyses Levinas's notion of subjectivity and examines its possible links with the notion developed by Kant, understood the latter as an exemplary vision of the modern explanation of subjectivity. Hofmeyr's position is discussed, who argues that there was a paradigmatic shift between an "early Levinas" centred on a freedom of Kantian type, and a "later Levinas" focused on passivity and that transcends Kant. The article shows that subjectivity in Levinas can never be understood as an activity in the Kantian sense.

Key words: Subjectivity; Levinas; Kant; alterity; activity-passivity; life of enjoyment; knowledge; substitution

\section{INTRODUCCIÓN}

Un concepto fundamental de la filosofía que surge bajo una nueva luz en el pensamiento de Emmanuel Levinas es la subjetividad. Según este autor, en la tradición moderna dicho concepto constituye una forma de egoísmo. Uno de los principales filósofos a los que Levinas asocia con esta noción de subjetividad es Kant. A primera vista, esto parecería ser inusual, pues Kant es el filósofo de la imparcialidad, la universalidad y un defensor del desinterés. Para Kant, la voluntad [Wille] que se configura en términos del interés propio no es moral, sino patológica. Él elabora un principio que sustenta la comprensión moderna de la moralidad: el concepto de libertad como autonomía. Para 
Levinas, dicha elaboración kantiana está en desacuerdo con su propia noción de pasividad ética (Levinas, 1978, pp. 99-129).

En este contexto, Daniel Smith afirma que la crítica de Levinas a Kant efectivamente da en el blanco: "si uno sigue la mecánica del relato de Kant, encontramos exactamente el tipo de caída en el egoísmo que esperaríamos encontrar, si el relato de Levinas de la historia de la filosofía es correcto"'(Smith, 2016, p. 3). No obstante, Gabriela Basterra afirma que la subjetividad de Kant se puede ver más cerca de la subjetividad de Levinas, en la medida en que ambos parecen concebir este concepto como "constituido por el impacto de un exceso [que] se calcula como un comando que ha sido recibido" (Basterra, 2015, p. 16). Varios pasajes de la filosofía moral de Kant parecen validar esta interpretación. Por ejemplo, cuando Kant escribe que "no queda, pues, otra cosa que pueda determinar la voluntad, si no es, objetivamente, la ley y, subjetivamente, el respeto puro a esa ley práctica" (Kant, 2005, p. 400). A partir de estas afirmaciones, Basterra sostiene que la subjetividad de Kant no es autonomía, sino heteronomía frente a la ley moral. En este contexto, la autora agrega que "la concepción de la ley moral que atraviesa la filosofía práctica de Kant tiene el rastro de la alteridad" (Basterra, 2015, p. 2). Si esta interpretación es correcta, entonces Kant y Levinas coinciden en el sentido de que la alteridad es el fundamento de la ética. Considerando sus interpretaciones, Smith y Basterra no están de acuerdo con sus lecturas de la relación Kant-Levinas. Sin embargo, el foco de estos autores es más bien Kant y no Levinas, pues dan por hecho que el segundo elabora un modelo ético centrado en la pasividad y desde ahí examinan si el primero puede ser leído o no en el mismo sentido. Por un lado, Smith sostiene que Kant no puede ser leído colocando al centro de la ética una concepción similar a la de Levinas. Por otro lado, para Basterra la filosofía de Kant puede dejar espacio para dicha interpretación.

Por el contrario, el foco de este artículo es distinto y puede leerse como sigue: ¿La noción de subjetividad de Levinas puede verse como un eco de la noción de autonomía de Kant? Esto porque, a pesar de la interpretación ampliamente reconocida que defiende que la noción de subjetividad de Levinas es completamente diferente a la de Kant (Davies, 2002;Chalier, 2002; Bernasconi, 2001, 2002), aún para algunos hay rastros de autonomía kantiana en el concepto de subjetividad de Levinas. Benda Hofyener ha señalado que existe un cambio paradigmático entre un Levinas temprano en Totalidad e infinito (y otros textos tempranos) y un Levinas posterior que se puede encontrar en De otro modo que ser (Hofyener, 2009, p. 21). El primero, aparentemente da prioridad al egoísmo y la libertad, en el sentido kantiano, y el segundo a la pasividad. En este contexto, Hofyener sostiene:

El pensamiento de Levinas nos ha dejado dos alternativas igualmente indeseables: elegir el yo y la libertad, lo que necesariamente equivale a un egoísmo absolutista en el esquema de las cosas de Levinas, o uno elige para el 
otro un altruismo aplastante en el que todos los deberes hacia el yo deben ser necesariamente abandonados (Hofyener, 2009, p. 28).

De manera similar, Surber y Crawford leyeron un momento de autonomía en la subjetividad de Levinas (Surber, 1994; Crawford, 2002). Frente a esta interpretación, el artículo muestra que la subjetividad ética en la filosofía de Levinas es esencialmente pasividad y nunca puede ser entendida en términos de autonomía en la tradición kantiana. Como ya se mencionó, esta lectura de Levinas es compartida por varios intérpretes. Sin embargo, esta interpretación emerge fundamentalmente desde las nociones de sustitución y asimetría que son parte del acervo del "Levinas posterior" que identifica Hofyener. En este contexto, el artículo examina el concepto de sustitución para mostrar dos cosas: por un lado, que efectivamente la ética en Levinas es pasividad. Por otro lado, para discutir posibles interpretaciones, a partir de estas Kant podría ser leído en el sentido de la pasividad. Ahora bien, el escrito muestra además que no solo en el "Levinas posterior", sino que también en el "Levinas temprano", la noción de subjetividad no puede ser igualada a la concepción kantiana. En eso radica uno de sus aportes centrales. Otro de sus contribuciones pasa por mostrar que en oposición a lo indicado por Hofyener, existe una relación de continuidad entre el "Levinas temprano" y el "Levinas posterior".

Para abordar la discusión propuesta, el texto se divide en las siguientes secciones. En la primera parte se discuten similitudes entre Kant y Levinas. Luego se elabora la tensión entre actividad y pasividad en Levinas, inicialmente en la noción de "vida del goce" (segunda sección), y posteriormente en el concepto de sustitución (tercera sección). Finalmente, en la conclusión se evalúa la noción de subjetividad ética en Levinas y se reflexiona acerca de su principal característica: la pasividad.

\section{SIMILITUDES ENTRE KANT Y LEVINAS}

En esta sección se discuten las principales similitudes entre Kant y Levinas, algunas de ellas destacadas en la literatura especializada (Atterton, 1999; Critchley, 2002; Cohen, 2006; Bernasconi, 2001, 2002; Bernet, 2002; Basterra, 2015; Chalier, 2002). El objetivo de esta presentación es constituir un primer escenario donde se vuelve posible hacer dialogar a estos filósofos. En ese contexto, una primera similitud es que ambos autores entienden la ética como el punto central de la filosofía y dan prioridad a la razón práctica (Atterton,1999; Critchley, 2002; Cohen, 2006). Kant afirma en el prefacio de la Crítica de la razón pura que la razón práctica es la pieza central de todo el edificio de la razón (5: 3). Esto se puede interpretar en el sentido de que la actividad del sujeto es lo que abre el mundo como un espacio donde pueden aparecer los objetos del conocimiento (Allison, 2004). Levinas también privilegia la ética sobre la racionalidad cognitiva, incluso en un sentido mucho más fuerte que Kant (Critchley, 2002; Cohen, 2006). En este punto, sin embargo, Levinas está más cerca de Kant que de otros filósofos como Descartes o Husserl, para quienes el dominio 
teórico tiene prioridad sobre lo ético. De hecho, en la tradición cartesiana, la epistemología es filosofía primera (Beiser, 2008). Por el contrario, en Levinas la ética es fundamental, es filosofía primera y no depende de la epistemología o la ontología (Levinas,1978; Bernasconi, 2001, 2002; Davies, 2002).

Una segunda similitud es que ambos pensadores consideran la subjetividad como un fenómeno que está en el centro de la filosofía. Es así como el proyecto mismo kantiano consiste en realizar una crítica de la racionalidad de la subjetividad. Como es sabido, la crítica de Kant a la razón no es destructiva, sino que busca dilucidar en detalle los límites de la razón y su posibilidad de concebir a priori los principios y leyes que regulan su propia agencia (tanto cognitiva, como práctica) (Allison, 2004). Por su parte, Levinas es un autor que desarrolla la noción de subjetividad, y se diferencia de la filosofía contemporánea que busca disolver el sujeto en las estructuras del lenguaje, en la acción comunicativa habermasiana o en la intersubjetividad hegeliana (Davies, 2002, p. 164). Sin embargo, no comprende la subjetividad "al nivel de su protesta puramente egoísta contra la totalidad, ni en su angustia ante la muerte, sino como fundada en la idea de lo infinito" (Levinas, 2002, p. 26). Más bien, su filosofía implica la transgresión de los límites de la conciencia a un sujeto abierto a la trascendencia del otro. A la luz de esta noción levinasiana de subjetividad, es posible delinear una primera diferencia con Kant. Por un lado, en Kant la subjetividad es la "condición para la representación de los seres" (Levinas, 1978, p. 179. Mi traducción) como representación y presencia (Heidegger, 1996; Allison, 2004). Por otro lado, en Levinas la subjetividad solo surge en el habla y el lenguaje, y la relación con el otro no puede entenderse como representación de la subjetividad de un objeto de la experiencia. Este problema está relacionado con sus diferentes nociones de temporalidad (Cohen, 2006). La subjetividad de Kant permanece dentro de los límites de un concepto de tiempo basado en la presencia, mientras que la subjetividad de Levinas supera esta concepción de temporalidad e invoca un tiempo fenomenológico. ${ }^{1}$

Una tercera similitud es que la comprensión de Levinas de la relación ética con el otro puede observarse como una reinterpretación de la segunda formulación de Kant del imperativo categórico, a saber, el reconocimiento de los otros como fines en sí mismos (Critchley, 2002, p. 429). Esta conexión fue observada por el mismo Levinas, quien señala que la primacía del encuentro cara a cara "es hacia una relación de este tipo que Kant acelera, cuando formula la segunda versión del imperativo categórico por una deducción, que puede ser válida o no, de la universalidad de la máxima" (Levinas, 1989, p. 146. Mi

\footnotetext{
${ }^{1}$ Aquí la crítica de Levinas a Kant sigue la reflexión de Ser y Tiempo de Heidegger (1996), véase el $\S 6$. La noción de temporalidad de Levinas, sin embargo, difiere de manera importante con el concepto de temporalidad de Heidegger (Cohen, 2006), así como también del concepto de temporalidad de Husserl (Bernet, 2002, pp. 82-99).
} 
traducción). Aquí, Levinas señala un problema difícil en la tradición kantiana: el éxito de Kant de derivar la fórmula de la humanidad del imperativo categórico (Allison, 1990). Según la literatura especializada, aunque la apelación al reconocimiento de la humanidad es atractiva per se, simplemente Kant es incapaz de derivar esta de la formulación del imperativo categórico, que permanece como una ley puramente formal y universal (Pinkard, 2002; Freyenhagen, 2011). Adicionalmente, aunque Kant reconoce una relación con el otro (en la fórmula de humanidad del imperativo), aún el punto de partida es la noción de autonomía. Por el contrario, en Levinas, el sujeto ético es pasividad radical frente al otro (Levinas, 1978).

Finalmente, una cuarta similitud es que tanto la ley moral en Kant como el infinito en Levinas son incomprensibles. Como señala Simon Critchley, "Kant concluye la Fundamentación [...] afirmando la incomprensibilidad de la ley moral" (2002, p. 12). Kant luego afirma en la Crítica de la Razón Práctica que la deducción de la ley moral es un "hecho de la razón". Por tanto, la moralidad kantiana parece concluir reconociendo la imposibilidad de asegurar la necesidad de la ley moral (Allison, 1990; Pinkard, 2002). Sin embargo, según Kant, comprendemos esta incomprensibilidad. Esto se asemeja a Levinas cuando afirma que "En la idea de lo infinito se piensa lo que permanece siempre exterior al pensamiento" (Levinas, 2002, p. 51). Este pensamiento se entiende como deseo metafísico y conduce a la exposición del sujeto al otro, una exposición que no puede entenderse como goce o conocimiento.

\section{ACTIVIDAD Y PASIVIDAD: LA VIDA DEL GOCE}

Según Hofyener (2009), en libros tempranos de Levinas (1987; 2001; 2002) la descripción del sujeto está ligada a su necesidad de escapar de sí mismo y de la pesadez de su propio ser. Esta es una experiencia fenomenológica que se manifiesta, por ejemplo, cuando el sujeto experimenta la angustia y la desesperación de la náusea como un antagonismo interno. Este peso del ser se resuelve en la relación ética, que se constituye en el encuentro con el otro. En este punto, Hofyener señala:

Levinas, por lo tanto, sostiene que el sujeto solo nace verdaderamente, en cualquier sentido significativo, como sujeto ético, es decir, después de que el Otro me ha "convertido" para enfrentar mi responsabilidad infinita hacia los demás (2009, p. 20).

Sin embargo, el sujeto también tiene una existencia económica, que Levinas llama la vida del goce [Vivre de jouissance], la que es pre-ética o egoísta. Dicho esto, la existencia económica es éticamente necesaria. Por tanto, en los trabajos "tempranos", la subjetividad puede entenderse de dos maneras. Por un lado, en la "vida del goce", la subjetividad se caracteriza como un yo egoísta independiente que encarna la libertad "arbitraria" de la existencia económica. Y se entiende como un ente sin oídos, más parecido a "el vientre 
hambriento que no tiene orejas" (Levinas, 2002, p.137). Esto significa que el sujeto tiende a persistir en la búsqueda ciega de sus impulsos y deseos egoístas (Hofyener, 2009, p. 20). Por otro lado, incluso en la "vida del goce", el sujeto está "obsesionado por una negatividad en el corazón de su existencia: el esfuerzo por evadir la gravedad de la materialidad y la soledad" (p. 20). Según Hofmeyr, en Totalidad e infinito esta "nada" abre una dimensión de interioridad en la subjetividad en donde podrá esperar y recibir la revelación de la trascendencia. Esta "frontera" no proviene de "la revelación del Otro... sino de la nada" (Hofyener, 2009, p. 20). En cualquier caso, en este segundo momento el sujeto es capaz de "autocrítica" y está abierto a la significación por medio del otro. Al unir las dos definiciones, entendemos que el sujeto es un existente sordo preocupado por su propia existencia, pero al mismo tiempo está abierto a la autocrítica, lo que hace que el llamado del otro sea audible.

Continuando con su lectura, Hofyener afirma que después de los textos considerados como tempranos, Levinas descarta la base existencial económica del sujeto que es fundamento de la relación ética. En De otro modo que ser, la sensibilidad frente al otro no surge de una dimensión interna de la autonomía, sino del otro mismo, de la trascendencia e infinitud que el otro pone a disposición del vínculo ético. Como afirma Hofyener, la relación cara a cara "no es una elección hecha desde una posición de libertad radical [...] sino la pasividad radical de un ser elegido" (p. 20). Debido a esto, Hofyener argumenta que existe un claro cambio paradigmático discernible entre Totalidad e infinito y De otro modo que ser. El cambio es desde el egoísmo y la libertad, hacia el sometimiento de la libertad en la relación frente al otro (p. 21).

Ahora bien, la pregunta aquí es la siguiente: cuando Kant habla de autonomía y Levinas de la autonomía pre-ética de la "vida del goce", ¿Se están refiriendo a objetos similares? En este punto Levinas significa una condición pre-ética, donde la relación con el otro no ha tenido lugar, pero es éticamente necesaria. Por el contrario, la libertad de Kant es inextricablemente moral y la autonomía es una voluntad cuyas elecciones están determinadas por su propia legalidad. En Kant, la libertad y el respeto por la ley describen la lucha entre las inclinaciones, intereses y deseos del sujeto, y lo que este debe hacer. En oposición, en Levinas la libertad pre-ética no puede ser reeducada ni coincidir con el respeto. Se define como la "vida del goce" en la que el sujeto se encuentra ocupado solo de su propia existencia.

Si Kant y Levinas significan cosas diferentes cuando el primero reconstruye la subjetividad como autonomía y el segundo la examina como una existencia económica preética, entonces la interpretación de Hofyener es errónea. Dicho esto, queda todavía abierta la pregunta si el sujeto de Levinas puede verse como actividad o autonomía en el momento en que se produce el encuentro con la otredad, es decir, cuando el sujeto alcanza la esfera de la ética. En la siguiente sección se discute ese punto argumentando que mediante el concepto de sustitución [Substitution], no cabe duda que la dimensión ética de la 
subjetividad en Levinas debe entenderse siempre como pasividad radical, y nunca como actividad en el sentido kantiano.

\section{PASIVIDAD RADICAL: LA SUSTITUCIÓN}

A la luz del concepto de sustitución [Substitution], el sujeto ético en Levinas surge como pasividad radical, y "este término se opone deliberada y necesariamente a una concepción kantiana de autonomía" (Davies, 2002, p. 164). Levinas rechaza la concepción moderna de la subjetividad que "consistía en perderse a sí mismo y volver a encontrarse para poseerse al mostrarse, proponerse como tema, exponerse en la verdad" (Levinas, 1978, p. 99. Mi traducción). En la conciencia tiene lugar la no aventura del ser, y esta es la posición principal de la tradición filosófica occidental. Ya en Totalidad e infinito, Levinas desarrolla una noción del otro que no implica que este sea capturado en la no aventura de la conciencia que define la visión tradicional de la subjetividad moderna. En este libro, el encuentro con el otro se debe entender desde la noción de infinito. En términos generales, infinito se refiere a una relación en la que el otro no puede reducirse a lo mismo, el otro escapa al poder del sujeto e incluso impacta la subjetividad como ningún otro objeto o fuerza.

Además, el otro tiene un papel fundamental en la formación del yo. En Levinas, el ego "no es un poder que se genera a sí mismo, ningún ego o moi se inaugura por sus propios actos" (Butler, 2005, p. 86). Según Levinas, el ego surge en un nivel preontológico. En otras palabras, es esencialmente un escenario más allá del ser [esencia]. Este espacio es preontológico, porque el mundo fenoménico de las personas se vuelve disponible solo después de que el ego se ha formado. El surgimiento de la subjetividad tiene lugar mediante el afecto del otro, el traumatismo anárquico de esta relación (Levinas, 1996, p. 93. Mi traducción). Este afecto es persecución, obsesión, y el objeto de esto es el sufrimiento del otro. El ego se define como proximidad fenomenológica y sensibilidad. El sujeto se ve afectado por el rostro del otro y este afecto es, en cierto modo, su condición de posibilidad. Por tanto, el ego aparece en la relación intersubjetiva.

Levinas describe este encuentro con el otro y argumenta que a partir del sufrimiento del sufrimiento (el sufrimiento por el sufrimiento del otro) se revela "una pasividad y asimetría en el corazón mismo de la subjetividad" (Davies, 2002, p.176). El yo es pasivo respecto de este sufrimiento, y esta persecución, pasión por el otro, toma la forma de sustitución: "algo se coloca en mi lugar, y emerge un Yo que puede entender su lugar de ninguna otra manera que este lugar ya ocupado por otro" (Butler, 2005, p. 89). La sustitución es un evento infinitamente recurrente, y esto significa que siempre está teniendo lugar. La referencia de Levinas a la formación del sujeto no tiene que ver con el desarrollo desde la niñez hasta la edad adulta, y por esta razón no pertenece a un proceso histórico (exposición diacrónica). Más bien, esta condición se entiende como un evento sincrónico e 
infinitamente recurrente, a partir de que es una relación con alguien que siempre ha estado allí. Levinas usa la figura del rehén para iluminar esta estructura:

El yo es un rehén porque no he hecho nada y siempre he estado acusado, perseguido. La ipseidad, en la pasividad sin una característica de identidad, es un rehén. La palabra Yo significa aquí estoy, respondiendo por todo y para todos (Levinas, 1978, p. 114. Mi traducción).

Mediante esta condición de ser un rehén, la ética es posible: permite la aparición de la piedad, la compasión, el perdón y la proximidad. El rehén es perseguido, obsesionado y pasivo, tiene que dar una respuesta antes de la responsabilidad de las responsabilidades del Otro y del Otro. Es por esto que Levinas afirma que "El yo es un sujeto bajo el peso del universo". Ser un rehén, "siempre es tener un grado de responsabilidad más, la responsabilidad de la responsabilidad del otro" (Levinas, 1978, p. 117. Mi traducción). Levinas no quiere decir que uno es responsable del otro como si uno fuera el autor de sus actos. La clave es que el sujeto afirma la falta de libertad en el centro de sus relaciones éticas. En este enfoque fenomenológico la relación con el otro es asimétrica y está más allá del libre albedrío, no ha sido elegida. En Levinas no hay autonomía, ni libertad, el sujeto está obsesionado, perseguido en el encuentro cara a cara. La experiencia de la responsabilidad no es la experiencia de una elección libre, sino más bien "la imposibilidad de evadir el llamado del vecino" (Levinas, 1996, p. 95. Mi traducción).

A la luz de esta noción de pasividad ética, existe otra posibilidad de examinar los temas éticos de Kant y Levinas que merecen atención, a saber, ¿En qué medida Kant puede dejar espacio para la pasividad? ¿Es posible argumentar una heteronomía inaugural acerca del respeto por la ley moral en Kant? (Basterra, 2015). En Kant el sujeto se da a sí mismo la ley moral, por lo tanto, es fundamentalmente autonomía. Dicho esto, Hofmeyr afirma que en el kantianismo el sujeto no puede establecer la ley por la cual descubre que es gobernado (p. 22). Por tanto, la ley moral de Kant podría ser una forma de pasividad en el sentido de Levinas. Además, en Kant, la ley moral parece ser una especie de dimensión nouménica inaccesible, "dentro del sujeto, [pero] paradójicamente ordenándola desde afuera, por así decirlo" (Hofyener, 2009, p. 22). Este artículo no está de acuerdo con esta interpretación de la filosofía moral de Kant. En el pensamiento de este autor, el sujeto es autónomo porque es una voluntad [Wille] que legisla normas universales desde el punto de vista moral. Como dice Davies, en Kant "la subjetividad es el respeto a la ley moral; libertad; espontaneidad; autonomía. Mientras tanto, en Levinas, transmite la responsabilidad por el otro; sinceridad; pasividad; separación; heteronomía" (Davies, 2002, p. 167). El núcleo de Levinas es la pasividad porque el vecino me asigna antes de designarlo. En Kant, el sujeto produce la ley moral, que por principio es imparcial y universal.

Mediante el concepto de sustitución, Levinas desarrolla una comprensión del sujeto radicalmente diferente al sujeto autónomo de Kant. En Levinas, el sujeto que está expuesto 92 | AlPHa No50 (Julio 2020) PÁGS. 85-96. ISSN 07 16-4254 
nunca puede permanecer en silencio y debe pensarse como "separación". Esta concepción de la subjetividad es fundamental para comprender la asimetría que Levinas establece en el centro de la relación ética. El sujeto en Levinas no es una función de su autonomía. Es esencialmente diferente al sujeto de Kant que debe abstraer deseos e inclinaciones para dar forma a la voluntad moral [Wille]. Además, la pasividad de Levinas en el centro de la relación ética implica que este origen, que está fuera del sujeto, nunca puede ser conocido como tal, no puede ser un tema de representación. El "yo pienso" como el fundamento de la moralidad, ya que el nacimiento del sujeto como una subjetividad libre, no es el comienzo de la vida ética en Levinas.

Según algunos comentaristas, "aunque la obligación precede a cualquier contrato o decisión consciente, no está totalmente fuera de nuestra libertad porque invita a nuestra libertad y nos convoca a la autolegislación frente a la otra" (Crawford, 2002, p. 515). Sin embargo, esto es incorrecto, pues si nos enfocamos en el concepto de sustitución, está claro que el otro toma mi lugar, por lo que la obligación ética nunca viene "de mí", sino siempre del Otro. El sujeto de Levinas no puede retratarse como un sujeto moral en un reino de fines en sí mismos que legislan normas universales (Kant, 2005, pp. 433, 446). Si ese fuera el caso, entonces la sustitución estaría basada en el "yo pienso" kantiano y no en el "aquí estoy" levinasiano.

\section{CONCLUSIÓN: LA SUBJETIVIDAD EN KANT Y EN LEVINAS}

En este artículo he argumentado que la filosofía ética de Levinas no coincide con la autonomía en el sentido kantiano, a diferencia de lo que afirman Hofyener y otros autores. La libertad pre-ética levinasiana no puede entenderse como un eco de la libertad de Kant. Esta interpretación sería errónea, pues en Kant la autonomía es esencialmente ética, mientras que en Levinas la libertad es pre-ética. La autonomía en Levinas no fundamenta la relación ética. Levinas es claro en este punto y descarta la interpretación que ve en la relación ética un momento de autonomía, por ejemplo, en su concepto de la "vida del goce". En este contexto, el filósofo francés señala: "el rostro me habla y por ello me invita a una relación sin paralelo con un poder que se ejerce, ya sea gozo o conocimiento" (Levinas, 2002, p. 211). Desde esto se concluye que el origen del sujeto "de ninguna manera puede estar ligado a la cognición" (Davies, 2002, p. 166). Consecuentemente, Kant y Levinas entienden la moral en sentidos diferentes. Es verdad que ambos filósofos hablan de la responsabilidad en la moralidad, pero lo que quieren decir no es lo mismo: mientras que en Kant la responsabilidad tiene como referencia la ley moral, en Levinas se enfrenta a la otredad.

$\mathrm{Si}$ una respuesta kantiana es un comienzo que surge del Yo pienso, de la espontaneidad y autonomía del sujeto; en Levinas lo que se descubre es una respuesta al "Aquí estoy" expresado por el Otro. Por tanto, en Levinas la pasividad radical fundamenta 
al sujeto ético. Esta pasividad en la relación con el Otro es una proximidad que no es una distancia geométrica, sino una obligación y un deseo previo a cualquier compromiso. Esto expresa una forma de ser afectado que de ninguna manera puede ser invertido por la espontaneidad de la subjetividad (Levinas, 1978, p. 100. Mi traducción). Por ello, el concepto de subjetividad de Levinas difiere de la noción de Kant del mismo concepto, básicamente porque en Levinas el sujeto no se ve como espontaneidad o actividad. En el mismo sentido de Davies, afirmamos que el proyecto filosófico levinasiano no es ni puede ser el proyecto de Kant (Davies, 2002, p. 165). En cambio, lo que ocurre en Levinas es la pasividad de un sujeto que se ve afectado por la llamada del otro.

La trascendencia de Levinas está expuesta en términos de responsabilidad y deseo metafísico. Como escribe Levinas, la trascendencia hacia el infinito tiene lugar en "el resplandor de la exterioridad o de la trascendencia en el rostro del otro" (Levinas, 2002, p. 51). Esta idea del infinito no tiene eco en el kantismo. Además, si el encuentro con el Otro implica la exterioridad del infinito, también determina la búsqueda para superar la carga del ser que pertenece a la subjetividad. Este motivo determina una mayor diferencia entre Levinas y Kant respecto de la ontología. La necesidad de escapar de la pesadez del ser es un motivo filosófico que ya estaba presente en los primeros libros de Levinas (2001). En este punto, el autor busca un pensamiento más allá de la ontología. Por el contrario, "No hay forma de salir de la reflexión kantiana sobre la subjetividad del espacio y el tiempo, hacia el sentido del sujeto 'fuera' de la ontología. Así, escribe Levinas, el kantianismo es la base de la filosofia, si la filosofía es ontología" (Davies, 2002, p. 165). En la filosofía de Kant, la ontología se fortalece, toma la forma de una Crítica de la razón que se orienta hacia la reconstrucción de las condiciones de posibilidad de la experiencia. El proyecto de Levinas busca alcanzar una filosofía distinta de la ontología, donde ni el ser del sujeto ni el del Otro se encuentren por medio de la conciencia. A partir de esto, se observa que la posición ética de Levinas no puede igualarse a la de Kant, a pesar de los intentos de algunos intérpretes de encontrar una relación directa en el concepto de subjetividad en ambos filósofos. Para finalizar, solo queda invitar a otros investigadores a explorar la crítica de Levinas a la ontología e indagar si efectivamente el kantismo está preso de ella. A modo de sugerencia, sería interesante conocer si las versiones contemporáneas de este tipo de filosofía todavía son objeto de este tipo de críticas desde la fenomenología y su vertiente deconstructiva.

\section{OBRAS CITADAS}

Allison, Henry (2004). Kant's Transcendental Idealism. New Haven: Yale University. Press. (1990). Kant's theory of freedom. Cambridge University Press. 
Atterton, Peter (1999). "The proximity between Levinas and Kant: the primacy of pure practical reason". The Eighteenth Century Vol. 40, No. 3, Emmanuel Levinas and the Eighteenth Century. 244-260.

Basterra, Gabriela (2015). The Subject of Freedom: Kant, Levinas. New York: Fordham University Press.

Beiser, Frederick (2008). German Idealism: The Struggle against Subjectivism 17811801. Harvard: University Press.

Bernasconi, Robert (2002). "What is the question to which "Substitution" is the answer?". En: The Cambridge Companion to Levinas. Ed. Simon Critchley \& Robert Bernasconi. Cambridge University Press.

— (2001). "Foreword". En: Levinas, E. Existence and Existents. Trans. Alphonso Lingis. Duquesne University Press.

Bernet, Rudolf (2002). "Levinas's critique of Husserl". En: The Cambridge Companion to Levinas. Ed. Simon Critchley \& Robert Bernasconi. Cambridge University Press.

Butler, Judith (2005). Given an Account of Oneself. Fordham University Press.

Chalier, Catherine (2002). What I Ought I to Do? Morality in Kant and Levinas. Trans. Marie Todd. Cornell University Press.

Cohen, Richard (2006). "Choosing and the choosen: Sartre and Levinas". Les Cahiers d'Études Lévinassiennes 5.

Crawford, Darin (2002). "The Fact of Reason and the Face of the Other: Autonomy, Constraint, and Rational Agency in Kant and Levinas". The Southern Journal of Philosophy. DOI: 10.1111/j.2041-6962.2002.tb01914.x.

Critchley, Simon (2002). "Introduction". En: The Cambridge Companion to Levinas. Ed. Simon Critchley \& Robert Bernasconi. Cambridge University Press.

Davies, Paul (2002). "Sincerity and the End of Theodicy". En: The Cambridge Companion to Levinas. Ed. Simon Critchley \& Robert Bernasconi. Cambridge University Press.

Freyenhagen, Fabian (2011). "Empty, Useless, and Dangerous? Recent Kantian Replies to the Empty Formalism Objection”. Bulletin of the Hegel Society of Great Britain, 63.

Heidegger, Martín (1996). Being and Time. New York: State University of New York Press. Hofyener, Benda (2009). “Radical Passivity: Ethical Problem or Solution?”. En: Radical Passivity: Rethinking Ethical Agency in Levinas. Ed. Brenda Hofyener. Springer.

Kant, Immanuel (2005). Fundamentación para una metafísica de las costumbres, trad. R. Aramayo, Madrid: Alianza, 2005.

(1996). Critique of Practical Reason, ed. and trans. Mary J. Gregor. Cambridge, Cambridge University Press. 
Levinas, Emmanuel (2002). Totalidad e Infinito. Ensayo sobre la exterioridad. Ediciones Sigueme, Salamanca.

— (2001). Existence and Existents. Trans. Alphonso Lingis. Duquesne University Press.

_- (1996). Basic Philosophical Writings. Bloomington, IN: Indiana University Press,

— (1989). Revelation in the Jewish Tradition. En: The Levinas Reader. Ed. Richard Cohen. Trans. Sarah Richmond. Cambridge: Basil Blackwell

_ (1987). Time and the Other. Trans. Richard Cohen. Duquesne University Press.

_ (1978). Otherwise than Being or Beyond Essence. Trans. Alphonso Lingis. Dordrecht and Boston, MA: Kluwer Academic Publishers.

Pinkard, Terry (2002). German Philosophy 1760-1860: The Legacy of Idealism. Cambridge University Press.

Smith, David (2016). ““'After you sir!”: Substitution in Kant and Levinas”. Journal of the British Society for Phenomenology. DOI: 10.1080/00071773.2016.1256602

Surber, Jean (1994). "Kant, Levinas, and the Thought of the "Other"'. Philosophy Today. 38 (3):294-316. 